(C) Elsevier Sequoia S.A., Lausanne - Printed in the Netherlands

\title{
ELECTROCHEMICAL AND MORPHOLOGICAL STUDIES OF COPPER ELECTRODEPOSITS DEPOSITED ONTO A COPPER (111) SUBSTRATE IN THE PRESENCE OF HALO-COMPOUNDS OF ACETIC ACID
}

\section{B. S. SHESHADRI and VASANTHA KOPPA}

Department of Chemistry, Central College, Bangalore University, Bangalore (India)

(Received September 11, 1978)

\section{Summary}

Halo-compounds of acetic acid present in small quantities in an acid copper sulphate bath have been found to modify the growth of copper deposits on a copper (111) substrate and to increase the cathodic polarization. At low d.c. densities a transition in the type of growth from triangular pyramids to twinned pyramids and finally to polycrystalline deposit was observed. At high d.c. densities triangular and hexagonal pyramids transformed to truncated hexagonal and twinned pyramids which subsequently changed to a polycrystalline deposit. These changes are attributed to adsorption of the halo-compounds of acetic acid on the substrate. The mechanism of the deposition process is discussed. Surface coverages of addition agents determined from exchange current density values were found to fit a Langmuir adsorption isotherm. The negative free energies of adsorption of the addition agents indicate strong adsorption and inhibition of the deposition process by halo-compounds of acetic acid.

\section{Introduction}

Addition agents in electroplating baths are known to have remarkable effects on the physical properties of the electrodeposits. Although extensive use is made of addition agents in plating baths, the study of the exact mechanisms of their action is still in its infancy. An appropriate approach to a proper understanding of the role of addition agents is to study the morphology of electrodeposits grown on single-crystal substrates with and without addition agents. Knowledge of the morphological features thus obtained in combination with the evaluation of electrode kinetic parameters such as $i_{0}, b, \beta$ etc. may give a fuller understanding of the effect of addition agents. Work on these lines has been carried out by several workers [ 1 - 15]. The effect of halo-compounds of acetic acid on the morphology and kinetics [16] of copper deposition on a copper (100) substrate has been investigated. 


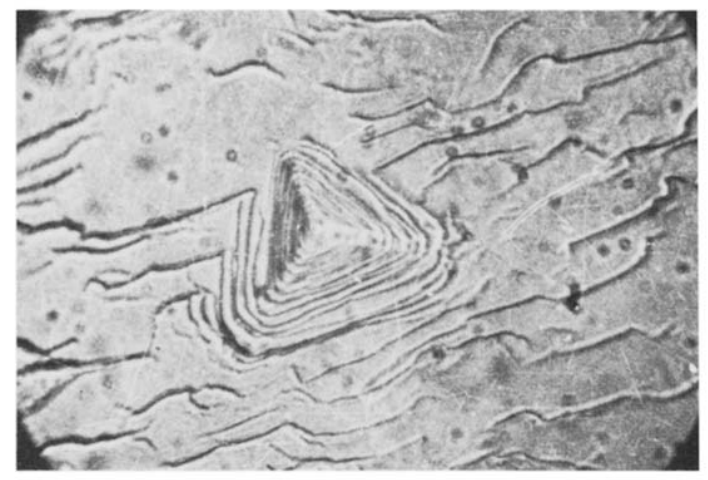

Fig. 1. Copper deposited on a copper (111) substrate from an acid copper sulphate bath at $5 \mathrm{~mA} \mathrm{~cm}^{-2}$.

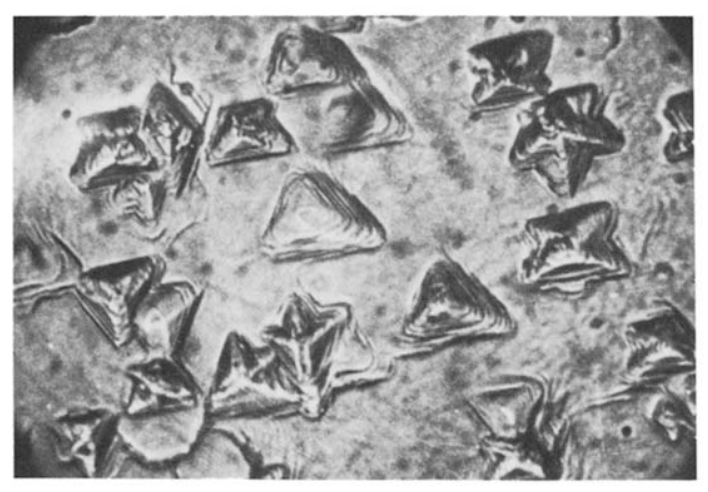

Fig. 2. Copper deposited on a copper (111) substrate from an acid copper sulphate bath $+10^{-4} \mathrm{M}$ chloroacetic acid at $5 \mathrm{~mA} \mathrm{~cm}^{-2}$.

Adsorption isotherms [17] for halo-compounds of acetic acid on a copper (100) substrate have also been reported. The object of the present work was to discover the effect of halo-compounds of acetic acid on the electrochemical and morphological properties of electrodeposited copper on a copper (111) substrate. We also report here the adsorption isotherms of halo-compounds of acetic acid on a copper (111) substrate during deposition.

\section{Experimental details}

AR grade copper sulphate was recrystallized three times using triply distilled water. The solution was subsequently pre-electrolysed, treated with carbon and filtered. Sulphuric acid (AR) was distilled once. A bath with the composition $0.25 \mathrm{M} \mathrm{CuSO}_{4}+0.1 \mathrm{M} \mathrm{H}_{2} \mathrm{SO}_{4}$ was prepared.

The copper (99.999\% purity) single-crystal (111) substrate was fixed in Tygon tubing so that only the desired plane was exposed. After the crystal 


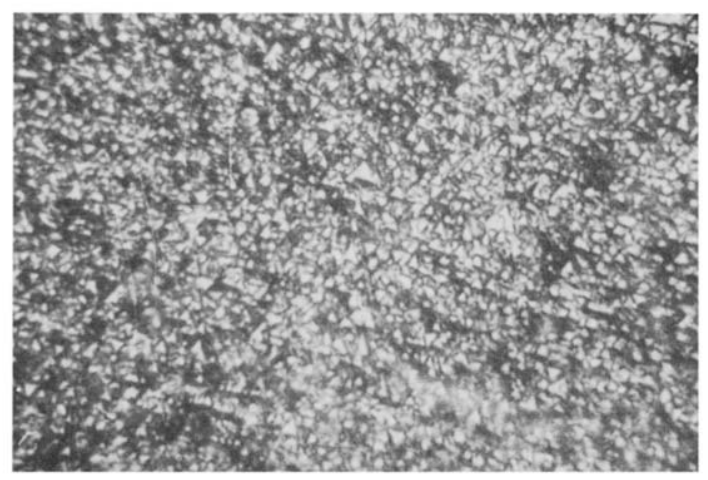

Fig. 3. Copper deposited on a copper (111) substrate from an acid copper sulphate bath $+2 \times 10^{-3} \mathrm{M}$ chloroacetic acid at $5 \mathrm{~mA} \mathrm{~cm}^{-2}$.

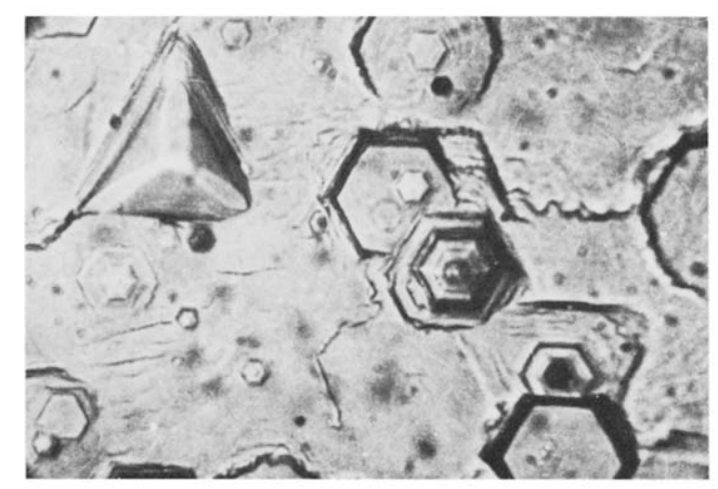

Fig. 4. Copper deposited on a copper (111) substrate from an acid copper sulphate bath at $10 \mathrm{~mA} \mathrm{~cm}^{-2}$.

had been mechanically polished on 4/0 emery paper using ethanol as lubricant, it was electropolished in 1:1 orthophosphoric acid [18] at a cell potential of $1.2 \mathrm{~V}$ for $30 \mathrm{~min}$ with a large copper cathode in an electropolishing cell. The crystal was removed without switching off the current and was washed with $10 \%$ phosphoric acid and then with conductivity water. It was immediately transferred to the deposition cell [19]. A polycrystalline copper foil (AR), the area of which was 40 times greater than that of the cathode, was used as an auxiliary electrode. A freshly prepared copper reference electrode was used for potential measurements during deposition.

A battery (about $90 \mathrm{~V}$ ) in series with a resistor was used as a source of constant current. The activation overpotential was recorded at regular intervals to an accuracy of $\pm 1 \mathrm{mV}$ using a high impedance voltmeter. At each current density the deposition was carried out up to a thickness of $10 \mathrm{C}$ $\mathrm{cm}^{-2}(3.6 \mu \mathrm{m})$. The surface of the deposit was examined with a microscope. All the micrographs were prepared at a magnification of $600 \times$. The experiments were repeated to ensure reproducibility. 


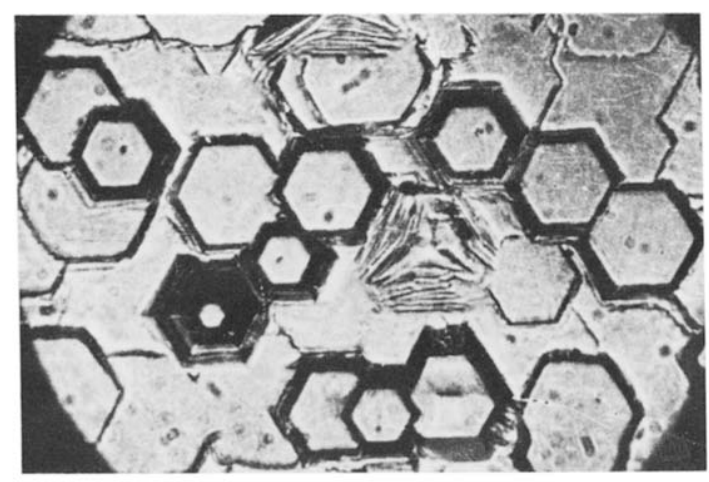

Fig. 5. Copper deposited on a copper (111) substrate from an acid copper sulphate bath $+10^{-4} \mathrm{M}$ chloroacetic acid at $10 \mathrm{~mA} \mathrm{~cm}^{-2}$.

\section{Results: morphology}

\subsection{Deposition in the presence of chloroacetic acid}

\subsubsection{At 2 and $5 \mathrm{~mA} \mathrm{~cm}^{-2}$}

Occasional triangular pyramids (Fig. 1) with steps on their sides appeared on a layered or smooth substrate at these current densities on the (111) substrate, as noticed by earlier workers [8] when copper was deposited from highly purified solution. The pyramidal faces were parallel to either the $\langle 112\rangle$ or the $\langle 110\rangle$ direction. When the chloroacetic acid concentration in the bath was varied from $10^{-10}$ to $5 \times 10^{-5} \mathrm{M}$ there was no appreciable change in the type of deposit. More and more triangular pyramids appeared with further gradual increase in the concentration. At $10^{-4} \mathrm{M}$ the entire surface of the crystal was covered with triangular pyramids (Fig. 2), some of which were twinned. When the concentration was increased further to $10^{-3} \mathrm{M}$, only twinned pyramids were observed. At $2 \times 10^{-3} \mathrm{M}$ only a polycrystalline deposit was observed (Fig. 3).

\subsubsection{At 10 and $15 \mathrm{~mA} \mathrm{~cm}^{-2}$}

Triangular and hexagonal pyramids (Fig. 4) appeared at these current densities when the copper was deposited from a highly purified acid copper sulphate bath. When the chloroacetic acid content in the bath was increased from $10^{-10}$ to $10^{-4} \mathrm{M}$ there was no change in the type of deposit except for an increase in the number of pyramids and a decrease in their size (Fig. 5). At the same time all the hexagonal pyramids truncated but the triangular ones did not. Both triangular pyramids and truncated hexagonal pyramids covered the surface completely at $5 \times 10^{-4} \mathrm{M}$. With further increase in the concentration of chloroacetic acid to $5 \times 10^{-3} \mathrm{M}$, the hexagonal pyramids transformed into blocks while a large number of small triangular pyramids, some of them twinned, were found (Fig. 6). At $7.5 \times 10^{-3} \mathrm{M}$ only triangular pyramids together with occasional hexagonal blocks appeared. At $10^{-2} \mathrm{M}$ chloroacetic acid only polycrystalline deposit was observed (cf. Fig. 3). 


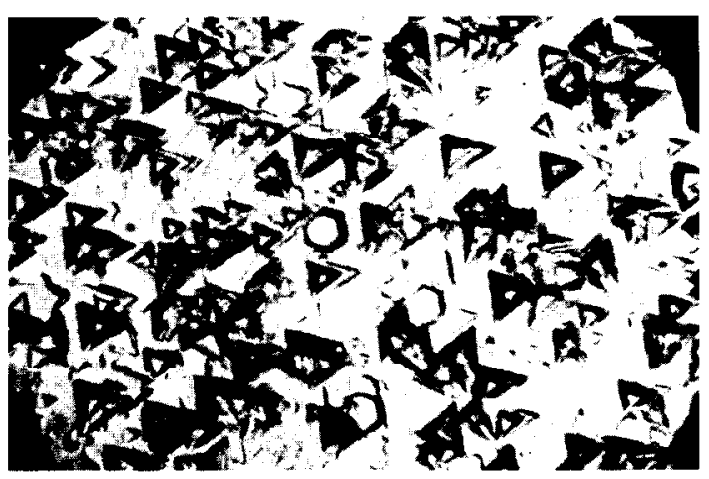

Fig. 6. Copper deposited on a copper (111) substrate from an acid copper sulphate bath $+5 \times 10^{-3} \mathrm{M}$ chloroacetic acid at $10 \mathrm{~mA} \mathrm{~cm}$.

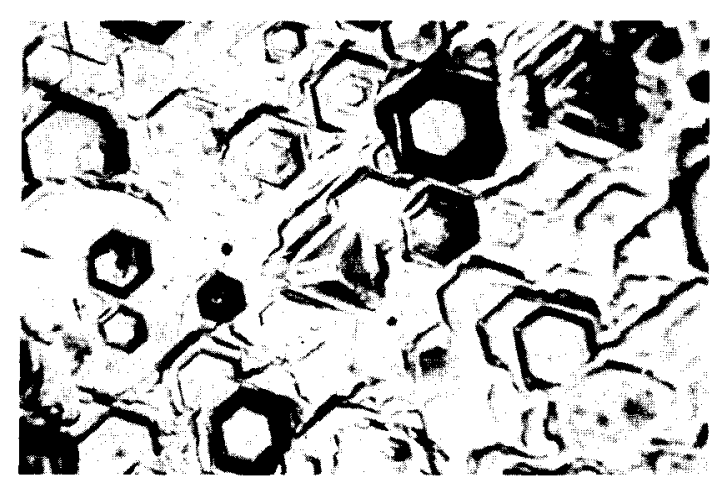

Fig. 7. Copper deposited on a copper (111) substrate from an acid copper sulphate bath $+10^{-5} \mathrm{M}$ bromoacetic acid at $5 \mathrm{~mA} \mathrm{~cm}^{-2}$.

Unlike at low current densities at a current density of $20 \mathrm{~mA} \mathrm{~cm}^{-2}$ no regular transition was observed in the presence of chloroacetic acid.

\subsection{Deposition in the presence of bromoacetic acid}

3.2.1. At 2 and $5 \mathrm{~mA} \mathrm{~cm}^{-2}$

When the concentration of bromoacetic acid in the bath was increased from $10^{-10}$ to $10^{-6} \mathrm{M}$ there was no change in the type of deposit obtained; only occasional triangular pyramids with steps appeared. At $5 \times 10^{-6} \mathrm{M}$ more small triangular pyramids were found. With further increase in the concentration to $10^{-5} \mathrm{M}$ hexagonal pyramids, some of which were truncated, appeared together with triangular pyramids (Fig. 7). At $10^{-4} \mathrm{M}$ a large number of small hexagonal pyramids together with occasional triangular ones (Fig. 8) were observed. The triangular pyramids disappeared completely and only a large number of small truncated hexagonal pyramids with blocks were noticed (Fig. 9) at a bromoacetic acid concentration of $10^{-3} \mathrm{M}$. With increase in the 


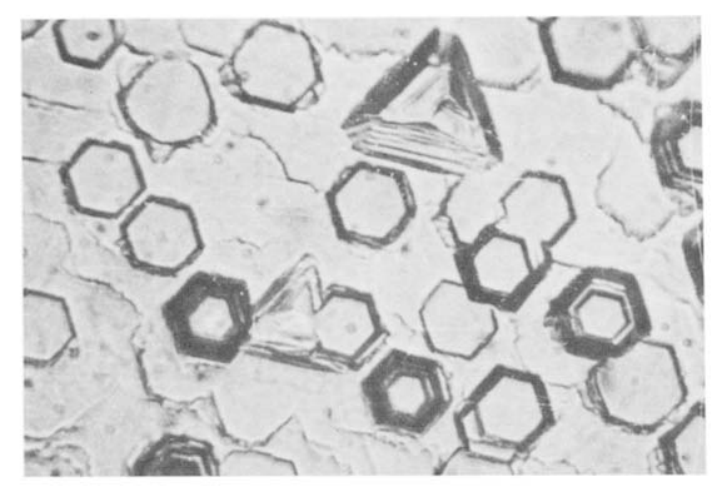

Fig. 8. Copper deposited on a copper (111) substrate from an acid copper sulphate bath $+10^{-4} \mathrm{M}$ bromoacetic acid at $5 \mathrm{~mA} \mathrm{~cm}^{-2}$.

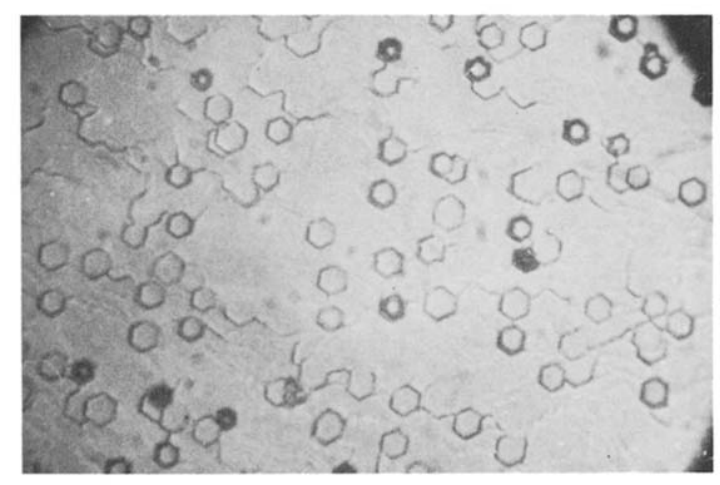

Fig. 9. Copper deposited on a copper (111) substrate from an acid copper sulphate bath $+10^{-3} \mathrm{M}$ bromoacetic acid at $5 \mathrm{~mA} \mathrm{~cm}^{-2}$.

concentration to $4 \times 10^{-3} \mathrm{M}$ the deposit gradually became polycrystalline (cf. Fig. 3).

\subsubsection{At 10 and $15 \mathrm{~mA} \mathrm{~cm}^{-2}$}

Triangular and hexagonal pyramids formed in pure solution gradually transformed to only the truncated hexagonal type at $5 \times 10^{-5} \mathrm{M}$ bromoacetic acid. More and more hexagonal pyramids nucleated with further increase in the concentration to $10^{-3} \mathrm{M}$. At $10^{-2} \mathrm{M}$ these pyramids gradually transformed to a polycrystalline type of deposit.

No regular transition in the type of deposit was observed when deposition was carried out at $20 \mathrm{~mA} \mathrm{~cm}^{-2}$ in the presence of bromoacetic acid.

\subsection{Deposition in the presence of iodoacetic acid}

\subsubsection{At 2 and $5 \mathrm{~mA} \mathrm{~cm}^{-2}$}

Triangular pyramids obtained under highly purified conditions at these current densities transformed completely to hexagonal pyramids (Fig. 10) 


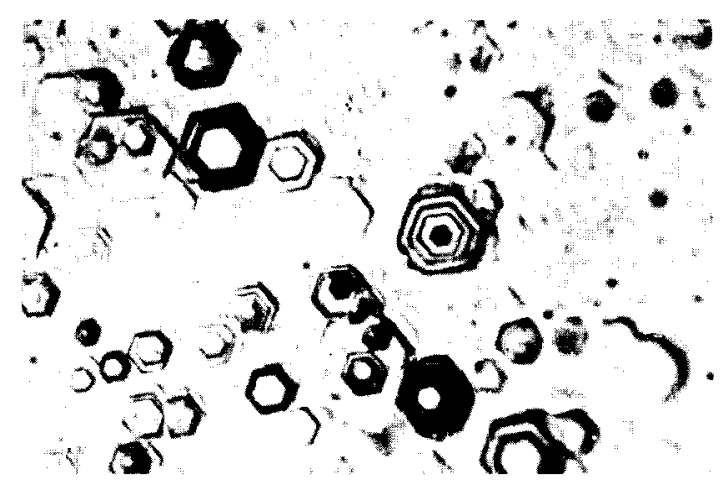

Fig. 10. Copper deposited on a copper (111) substrate from an acid copper sulphate bath $+10^{-6} \mathrm{M}$ iodoacetic acid at $5 \mathrm{~mA} \mathrm{~cm}^{-2}$.

when the concentration of iodoacetic acid in the bath was gradually increased from $10^{-8}$ to $10^{-6} \mathrm{M}$. At $5 \times 10^{-6} \mathrm{M}$ these hexagonal pyramids became truncated. Progressively more of these small hexagonal pyramids completely covered the surface at $10^{-5} \mathrm{M}$ iodoacetic acid. At $5 \times 10^{-5} \mathrm{M}$ they gradually changed to form a polycrystalline deposit.

\subsubsection{At 10 and $15 \mathrm{~mA} \mathrm{~cm}^{-2}$}

At $5 \times 10^{-6} \mathrm{M}$ iodoacetic acid only hexagonal pyramids appeared; they were small and numerous. When the concentration was increased to $5 \times$ $10^{-5} \mathrm{M}$ the pyramids became twinned and they covered the whole surface of the crystal. With further increase in the concentration more twinned pyramids occurred. They finally transformed to a polycrystalline deposit at a concentration of $10^{-4} \mathrm{M}$.

Deposition at $20 \mathrm{~mA} \mathrm{~cm}^{-2}$ in the presence of iodoacetic acid did not produce any gradual change in morphological features on the copper (111) substrate.

\section{Overpotentials}

On a copper (111) substrate the overpotential during deposition from a highly purified acid copper sulphate bath decreased with time at all current densities and attained a steady value [8]. The steady state was reached at more or less the same thickness of deposit for all current densities (Fig. 11). The trend in the variation of overpotential with time during deposition in the presence of a halo-compound of acetic acid was the same as that for the pure solution. However, in the presence of halo-compounds of acetic acid at effective concentrations which alter the surface topography of the deposit, overpotential values were always higher for both the initial and the final stages of deposition at any given current density. The overpotential attained a steady state at the same thickness of deposit in the presence of 

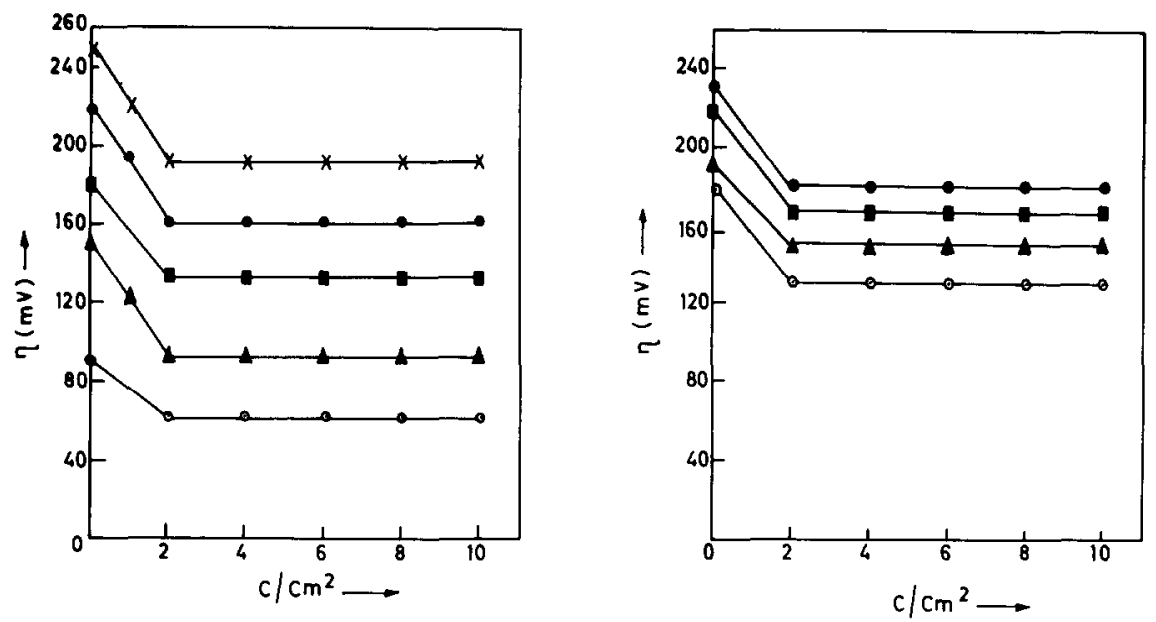

Fig. 11. The variation of overpotential with thickness of the deposit during deposition of copper from an acid copper sulphate bath at various current densities: $0,2 \mathrm{~mA} \mathrm{~cm}^{-2}$; $4,5 \mathrm{~mA} \mathrm{~cm}^{-2} ; \bullet, 10 \mathrm{~mA} \mathrm{~cm}{ }^{-2} ; \bullet, 15 \mathrm{~mA} \mathrm{~cm}^{-2} ; \times, 20 \mathrm{~mA} \mathrm{~cm}^{-2}$.

Fig. 12. The variation of overpotential with thickness of the deposit during deposition of copper from an acid copper sulphate bath at $10 \mathrm{~mA} \mathrm{~cm}^{-2}$ and at various chloroacetic acid concentrations: $O$, pure solution; $\Delta, 10^{-4} \mathrm{M} ; \mathrm{E}, 10^{-3} \mathrm{M} ; \bullet, 10^{-2} \mathrm{M}$.

halo-compounds of acetic acid. At any given current density the values of overpotentials in the presence of a halo-compound of acetic acid are in the order

$$
\eta(\text { chloroacetic })<\eta(\text { bromoacetic })<\eta(\text { iodoacetic })
$$

The overpotentials observed during deposition onto a (111) substrate at various concentrations of halo-compounds of acetic acid are shown in Figs. $12-14$, where a current density of $10 \mathrm{~m} \mathrm{~A} \mathrm{~cm}^{-2}$ has been chosen as typical. The figures indicate the above order of overpotential at any given concentration of halo-compounds of acetic acid at the given current density of 10 $\mathrm{mA} \mathrm{cm}{ }^{-2}$.

Tafel plots were constructed from the overpotential $\eta$ versus logarithm of current density $i$ dependence on a copper (111) substrate in pure solution as well as in the presence of halo-compounds of acetic acid. Exchange current densities $i_{0}$ were evaluated from the Tafel plots. In pure solution $i_{0}$ was $2.5 \mathrm{~mA} \mathrm{~cm}^{-2}$ on the (111) substrate with a Tafel slope $b$ of $125 \pm 5 \mathrm{mV}$. The values of exchange current densities $i_{0} *$ at various concentrations of halo-compounds of acetic acid are shown in Table 1.

It was found that the addition of a halo-compound of acetic acid produced a significant effect on $i_{0}$. The $i_{0}$ values gradually decreased with increase in the concentration of the halo-compound of acetic acid. Iodoacetic acid had a considerable effect on $i_{0}$ at low concentrations compared with chloroacetic acid and bromoacetic acid. 

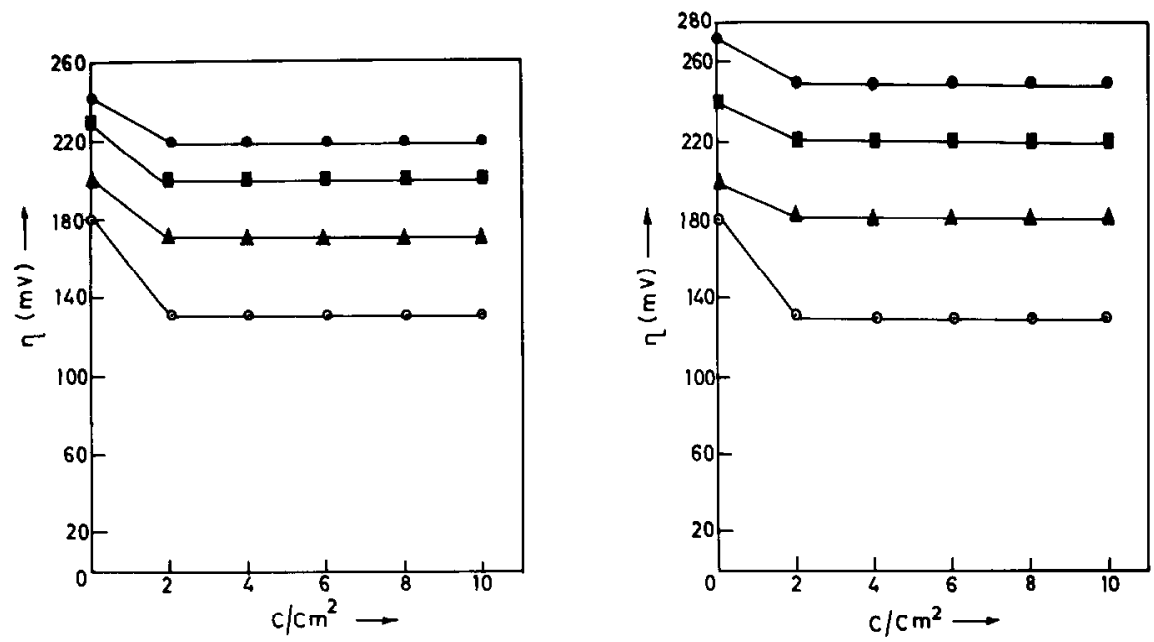

Fig. 13. The variation of overpotential with thickness of the deposit during deposition of copper from an acid copper sulphate bath at $10 \mathrm{~mA} \mathrm{~cm}$ and at various bromoacetic acid concentrations : $O$, pure solution; $\wedge, 10^{-4} \mathrm{M} ; \bullet, 10^{-3} \mathrm{M} ; \bullet, 10^{-2} \mathrm{M}$.

Fig. 14. The variation of overpotential with thickness of the deposit during deposition of copper from an acid copper sulphate bath at $10 \mathrm{~m} \mathrm{~A} \mathrm{~cm}^{-2}$ and at various iodoacetic acid concentrations: $O$, pure solution; $\wedge, 10^{-6} \mathrm{M} ; \bullet, 10^{-5} \mathrm{M} ; \bullet, 10^{-4} \mathrm{M}$.

\section{TABLE 1}

Exchange current density $i_{0}{ }^{*}$ on a copper (111) substrate in the presence of halo-compounds of acetic acid at $25{ }^{\circ} \mathrm{C}$.

\begin{tabular}{|c|c|c|c|}
\hline \multirow[t]{2}{*}{ Concentration (M) } & \multicolumn{3}{|l|}{$i_{0} *\left(\mathrm{~mA} \mathrm{~cm}^{-2}\right)$} \\
\hline & Chloroacetic acid & Bromoacetic acid & Iodoacetic acid \\
\hline $10^{-8}$ & - & - & 2.3 \\
\hline $10^{-7}$ & - & - & 1.8 \\
\hline $10^{-6}$ & 2.2 & 2.1 & 1.6 \\
\hline $10^{-5}$ & 1.8 & 1.7 & 1.2 \\
\hline $10^{-4}$ & 1.5 & 1.4 & 1.0 \\
\hline $10^{-3}$ & 1.3 & 1.2 & - \\
\hline $10^{-2}$ & 1.15 & 1.1 & - \\
\hline
\end{tabular}

The exchange current density has been shown [20] to be related to the rate of deposition. The surface coverages $\theta$ of the addition agents on the copper (111) substrate were evaluated using the equation [21]

$$
\theta=1-\frac{i_{0} *}{i_{0}}
$$

where $i_{0} *$ and $i_{0}$ represent the exchange current densities with and without addition agents respectively. The surface coverage of halo-compounds of 


\section{TABLE 2}

Surface coverage $\theta$ of halo-compounds of acetic acid on a copper (111) substrate in 0.25 $\mathrm{M} \mathrm{CuSO}_{4}+0.1 \mathrm{M} \mathrm{H}_{2} \mathrm{SO}_{4}$ at $25^{\circ} \mathrm{C}$

\begin{tabular}{llll}
\hline Concentration (M) & \multicolumn{3}{l}{$\theta$} \\
\cline { 2 - 4 } & Chloroacetic acid & Bromoacetic acid & Iodoacetic acid \\
\hline $10^{-8}$ & - & - & 0.08 \\
$10^{-7}$ & - & - & 0.28 \\
$10^{-6}$ & 0.12 & 0.16 & 0.36 \\
$10^{-5}$ & 0.28 & 0.32 & 0.52 \\
$10^{-4}$ & 0.41 & 0.43 & 0.6 \\
$10^{-3}$ & 0.48 & 0.52 & - \\
$10^{-2}$ & 0.54 & 0.56 & - \\
\hline
\end{tabular}

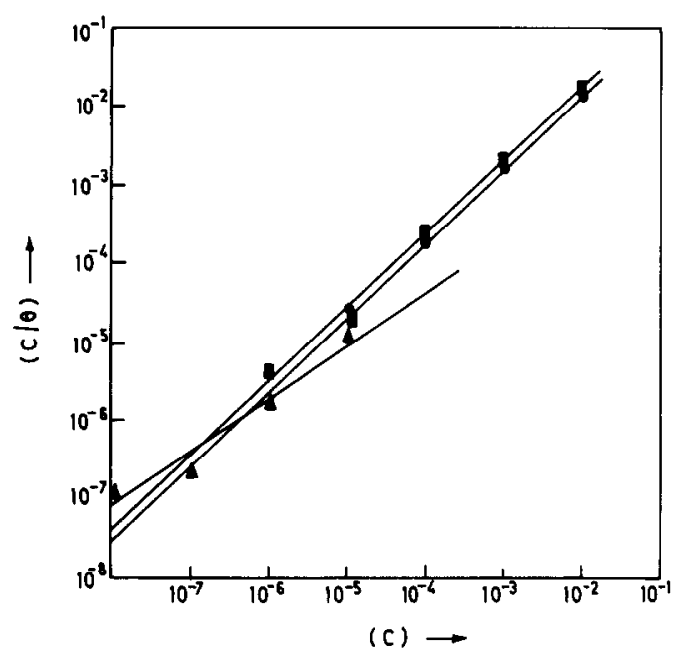

Fig. 15. Adsorption isotherms for the adsorption for halo-compounds of acetic acid on a copper (111) substrate in an acid copper sulphate bath at $25^{\circ} \mathrm{C}: \bullet$, chloroacetic acid; -, bromoacetic acid; $\mathbf{\Lambda}$, iodoacetic acid.

acetic acid on the (111) substrate at various concentrations is given in Table 2. A plot of $C / \theta$ against $C$ ( $C$ is the concentration of addition agent in the bulk) was made for the copper (111) substrate at $25^{\circ} \mathrm{C}$ for halo-compounds of acetic acid (Fig. 15).

The apparent free energies of adsorption $\Delta G_{\mathrm{a}}^{0}$ of halo-compounds of acetic acid at various concentrations on a copper (111) substrate were evaluated using the equation [22, 23]

$$
\Delta G_{\mathrm{a}}^{\mathbf{0}}=-2.303 R T \log \left[\frac{55.4 \theta}{C_{\text {org }}(1-\theta)^{x}} \frac{\{\theta+x(1-\theta)\}^{x-1}}{x^{x}}\right]
$$

where $C_{\text {org }}$ is the concentration of addition agent in the bulk and $x$ is the size factor. Using the values of molecular weights, densities and molecular radii 
TABLE 3

Apparent free energy of adsorption of halo-compounds of acetic acid at various surface coverages on a copper (111) substrate at $25{ }^{\circ} \mathrm{C}$

\begin{tabular}{|c|c|c|c|c|c|}
\hline \multicolumn{2}{|c|}{ Chloroacetic acid } & \multicolumn{2}{|c|}{ Bromoacetic acid } & \multicolumn{2}{|c|}{ Iodoacetic acid } \\
\hline$\theta$ & $\begin{array}{l}\Delta G_{\mathrm{a}}^{0} \\
\left(\mathrm{kcal} \mathrm{mol}^{-1} \text { ) }\right.\end{array}$ & $\theta$ & $\begin{array}{l}\Delta G_{\mathrm{a}}^{0} \\
\left(\mathrm{kcal} \mathrm{mol}^{-1}\right)\end{array}$ & $\theta$ & 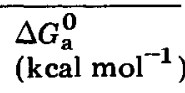 \\
\hline 0.12 & -8.65 & 0.16 & -8.806 & 0.08 & -10.8649 \\
\hline 0.28 & -8.0978 & 0.32 & -8.097 & 0.28 & -10.531 \\
\hline 0.41 & -6.5800 & 0.43 & -7.124 & 0.36 & -9.501 \\
\hline 0.48 & -5.998 & 0.52 & -6.0985 & 0.52 & -8.7054 \\
\hline 0.54 & -4.859 & 0.56 & -4.9008 & 0.60 & -7.6982 \\
\hline
\end{tabular}

of water and chloroacetic, bromoacetic and iodoacetic acids, the $x$ values were found to be $3.6,4$ and 5.2 respectively. The free energies of adsorption of halo-compounds of acetic acid were found to be negative (Table 3).

\section{Discussion}

On a copper (111) substrate the formation of triangular and hexagonal pyramids from a highly purified acid copper sulphate bath can be accounted for by the screw dislocation mechanism of growth. It is known [24] that the formation of pyramids is favoured by a mechanism involving a pair of screw dislocations of opposite sign. Macrosteps observed on the side faces of pyramids are formed by initial bunching of small ideally monatomic steps which originate at the apex of pyramids. The bunching mechanism is supported by the fact that there are no visible steps close to the apex. This is in agreement with the observations of earlier workers $[25,26]$.

It was observed that halo-compounds of acetic acid in an acidified copper sulphate bath altered the deposit morphology and increased the cathodic polarization. The Tafel slope was found to be $125 \pm 5 \mathrm{mV}$ when copper was deposited from an acidified copper sulphate bath. However, in the presence of halo-compounds of acetic acid the Tafel slope did not alter but there was a gradual decrease in the value of the exchange current density with increase in the concentration of the halo-compound of acetic acid.

The halo-compounds of acetic acid do not dissociate much in a strong acid medium. Therefore only molecules are adsorbed on the substrate surface. The molecules on the surface may form (a) an obstacle for the diffusion of adions to the growth sites or (b) a very loose complex with copper ions. These complex molecules may be adsorbed on the surface.

A larger number of triangular pyramids are found at low d.c. densities in the presence of halo-compounds of acetic acid. This increase in nucleation is due to the fact that there is an increase in deposition overpotential. With an increase in the concentration of the halo-compound of acetic acid more 
of the surface area of the copper (111) substrate is covered. The current density for the remaining surface area therefore increases, which increases the deposition overpotential. A higher deposition overpotential will lead to increased nucleation; hence twinning of pyramids occurs. Subsequently, at a critical concentration of the halo-compound of acetic acid, random nucleation occurs on an adsorbed film of the halo-compound of acetic acid, resulting in the formation of a polycrystalline deposit.

At higher current densities, in addition to increased nucleation in the presence of a halo-compound of acetic acid, both triangular and hexagonal pyramids become truncated. This may be because adsorption of the addition agents at the apex of pyramids prevents the generation of steps from the apex so that vertical growth of pyramids stops. The pyramids then gradually truncate. Truncated pyramids transform to blocks at appreciable concentrations of a halo-compound of acetic acid. At such concentrations of addition agent we would expect a larger surface area to be covered. Under these conditions the current density for the given area becomes considerably larger and hence results in a larger deposition overpotential. This enhances nucleation, producing twinned growth of pyramids and random nucleation (at a critical concentration of addition agent). The increase in deposition overpotential is shown to be dependent on the concentration of the halo-compound of acetic acid, as shown in Figs. 12 - 14 and 16.

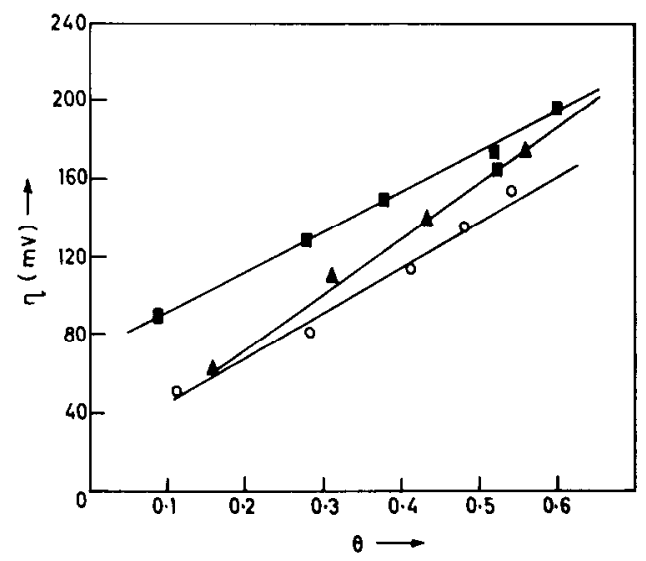

Fig. 16. The variation of overpotential with surface coverage of halo-compounds of acetic acid at $25^{\circ} \mathrm{C}: 0$, chloroacetic acid; $\wedge$, bromoacetic acid; $\boldsymbol{m}$, iodoacetic acid.

The variation in overpotential with deposition time on a copper (111) substrate has been explained by Damjanovic et al. [8]. They have proposed an empirical equation to account for the decrease in overpotential with deposition time and have also shown that at any given current density the overpotential changes markedly as the crystal grows. This they have attri- 
buted to the activity of the surface which depends strongly on the growth forms which develop during the deposition process.

The mechanism of deposition of copper from an acid copper sulphate bath is [2]

$$
\begin{aligned}
& \mathrm{Cu}^{2+}+\mathrm{e} \stackrel{\text { r.d.s. }}{\longrightarrow} \mathrm{Cu}^{+} \\
& \mathrm{Cu}^{+}+\mathrm{e} \longrightarrow \mathrm{Cu}
\end{aligned}
$$

The Tafel slope values of $125 \pm 5 \mathrm{mV}$ obtained with and without the addition of halo-compounds of acetic acid on a copper (111) substrate indicate that the mechanism of the first electron transfer reaction is the rate-determining step (r.d.s.).

The deposition process is known to begin at growth sites such as dislocations on the surface, misorientations and/or kinks in a step. The exchange current density is a measure of deposition rate which in turn depends on the number of growth sites [8]. Addition agents have been shown [21] to act as inhibitors for the deposition process through specific adsorption on the metal surface. With these facts taken into consideration and on the assumption that the adsorbed inhibitor molecules do not interact (at low values of surface coverage $\theta$ ), the apparent rate of deposition in the presence of inhibitor is proportional to the number of growth sites uncovered by the adsorbed molecules [21] .

By utilizing the concept of the Langmuir adsorption isotherm [27] a correlation between $\theta$ and $C$ can be achieved:

$$
\theta=K C /(1+K C)
$$

On rearranging eqn. 4 we obtain

$$
\frac{C}{\theta}=\frac{1}{K}+C
$$

The variation of $C / \theta$ with $C$ (Fig. 15) shows excellent agreement of the experimental results for the adsorption of halo-compounds of acetic acid with the Langmuir adsorption isotherm. The values of the constant $K$ of the Langmuir adsorption isotherm are listed in Table 4 for halo-compounds

\section{TABLE 4}

Values of the constant $K$ in the Langmuir isotherm at $25^{\circ} \mathrm{C}$

\begin{tabular}{ll}
\hline Addition agents & $K\left(\mathrm{~mol}^{-1}\right)$ \\
\hline Chloroacetic acid & $2 \times 10^{7}$ \\
Bromoacetic & $1.538 \times 10^{7}$ \\
Iodoacetic acid & $1.111 \times 10^{7}$ \\
\hline
\end{tabular}


of acetic acid, which are strongly adsorbed on the copper (111) substrate during the deposition process.

Electrosorption always involves displacement of solvent molecules at the interface [28]. The exchange of adsorbate between the bulk and the interface depends on the relative sizes of the adsorbate and the solvent [29]. This competitive adsorption of inhibitor molecules from solution is identical with the general quasi-chemical substitution process [22].

$$
\begin{aligned}
\text { inhibitor (solution) }+x \mathrm{H}_{2} \mathrm{O} \text { (adsorbed) }= & \text { inhibitor (adsorbed) }+ \\
& +x \mathrm{H}_{2} \mathrm{O} \text { (solution) }
\end{aligned}
$$

The free energies of adsorption of halo-compounds of acetic acid at any given concentration are negative on the copper (111) substrate. Hence it is expected that there is spontaneous adsorption and inhibition by these halo-compounds of acetic acid during the deposition process.

The above results indicate that there is a decrease in the rate of deposition in the presence of addition agents. This is due to the fact that the surface diffusion of adions to the growth sites is hindered or retarded [21] owing to the adsorbed film of the addition agent. The addition agents which retard the deposition rate are called inhibitors. Inhibitors thus increase the deposition overpotential ( $c f$. Figs. 12 - 14). The extent of these effects is dependent on the degree of surface coverage resulting from the inhibitor adsorptives. This is supported by the experimental result (Fig. 16) that the deposition overpotential increases linearly with $\theta$. As pointed out by Fischer [21], the halo-compounds of acetic acid exhibit the characteristic features of interfacial inhibitors.

It may be concluded that halo-compounds of acetic acid alter the deposit morphology on a copper (111) substrate and affect the deposition rate as well. This is attributed to the adsorption of halo-compounds of acetic acid on the substrate.

\section{Acknowledgments}

The authors would like to thank Professor G. K. Narayana Reddy, Head of the Department of Chemistry, Bangalore University, Bangalore for providing laboratory facilities and encouragement and Dr. S. M. Mayanna for helpful discussion. One of us (V.K.) thanks CSIR for a pool position.

\section{References}

1 B. E. Conway and J. O'M. Bockris, Proc. R. Soc., 248 (1958) 394.

2 E. Mattson and J. O M Bockris, Trans. Faraday Soc., 55 (1959) 1586.

3 S. C. Barnes, J. Electrochem. Soc., 111 (1964) 296.

4 D. R. Turner and G. R. Johnson, J. Electrochem. Soc., 109 (1962) 107, 178.

5 S. C. Barnes, Electrochim. Acta, 5 (1961) 79. 
6 S. C. Barnes and G. G. Storey, J. Electrochem. Soc., 106 (1959) 1081.

7 A. Damjanovic, M. Paonovic and J. O'M Bockris, J. Electroanal. Chem., 9 (1965) 93.

8 A. Damjanovic, T. H. V. Setty and J. OM. Bockris, J. Electrochem. Soc., 113 (1966) 429.

9 L. L. Shreir and J. W. Smith, Trans. Faraday Soc., 50 (1954) 393.

10 B. S. Sheshadri and T. H. V. Setty, Indian J. Chem., 8 (1970) 162.

11 B. S. Sheshadri and T. H. V. Setty, Trans. Soc. Adv. Electrochem. Sci. Technol., 7 (1972) 91.

12 B. S. Sheshadri and T. H. V. Setty, Proc. Indian Acad. Sci., Sect. A, 76 (4) (1972) 167.

13 B. S. Sheshadri and T. H. V. Setty, Indian J. Chem., 11 (1973) 927.

14 B. S. Sheshadri and T. H. V. Setty, Electrochim. Acta, 17 (1972) 1895.

15 B. S. Sheshadri, J. Electroanal. Chem. Interfacial Electrochem., 61 (1975) 353.

16 B. S. Sheshadri and T. H. V. Setty, Indian J. Technol., 11 (1973) 388.

17 B. S. Sheshadri and S. M. Mayanna, Electrodeposition Surf. Treat., 3 (1975) 189.

18 P. A. Jacquet, Met. Rev., 1 (1956) 157.

19 S. Nageswar and T. H. V. Setty, Proc. Indian Acad. Sci., Sect. A, 67 (1968) 178.

20 J. Tafel, Z. Phys. Chem. (Leipzig), 54 (1905) 641.

21 H. Fischer, Electrodeposition Surf. Treat., 1 (3) (1973) 239.

22 J. O'M. Bockris and D. A. J. Swinkells, J. Flectrochem. Soc., 111 (1964) 736.

23 S. M. Mayanna, Curr. Sci., 43 (1974) 746.

24 A. Damjanovic and J. O M. Bockris, in J. O'M. Bockris and B. E. Conway (eds.), Modern Aspects of Electrochemistry, Butterworths, London, 1964, p. 224.

25 N. Underwood, Phys. Rev., 47 (1935) 502.

26 F. C. Frank, Growth and Perfection of Crystals, Wiley, New York, 1958, p. 411.

27 S. M. Mayanna, J. Electrochem. Soc., 122 (1975) 251.

28 M. A. V. Devanathan, J. O'M. Bockris and K. Muller, Pruc. Roy. Suc. Londun, Ser. A, 274 (1963) 55.

29 H. P. Dhar, B. E. Conway and K. M. Joshi, Electrochim. Acta, 18 (1973) 789. 